\title{
Multi-Coupled Resonator Microwave Diplexer with High Isolation
}

\author{
Augustine O. Nwajana, Kenneth S. K. Yeo \\ Department of Electrical and Electronic Engineering \\ University of East London \\ London, UK \\ a.nwajana@ieee.org
}

\begin{abstract}
A microwave diplexer achieved by coupling a dualband bandpass filter onto two single-bands (transmit, $T x$ and receive, $R x$ ) bandpass filters is presented. This design eliminates the need for employing external junctions in diplexer design, as opposed to the conventional design approach which requires separate junctions for energy distribution. A 10-pole $\left(1^{\text {th }}\right.$ order $)$ diplexer has been successfully designed, simulated, fabricated and measured. The diplexer is composed of 2 poles from the dualband filter, 4 poles from the $T x$ bandpass filter, and the remaining 4 poles from the $R x$ bandpass filter. The design was implemented using asynchronously tuned microstrip square open-loop resonators. The simulation and measurement results show that an isolation of $50 \mathrm{~dB}$ is achieved between the diplexer $T x$ and $R x$ bands. The minimum insertion loss is $2.88 \mathrm{~dB}$ for the transmit band, and $2.95 \mathrm{~dB}$ for the receive band.
\end{abstract}

Keywords—diplexer; filters; coupling; microstrip; resonators

\section{INTRODUCTION}

Diplexer is device used for either splitting a frequency band into two sub-bands or for combining two sub-bands into one wide band [1]. It is popularly used in satellite communication systems to combine both the Tx and the Rx antennas on space crafts. Microwave diplexer is commonly used in the Radio Frequency (RF) front end of cellular radio base stations to separate the Tx and the Rx channels as shown in Fig. 1.

Conventionally, microwave diplexers are achieved by connecting two separately designed filters together via an external energy distribution device. This connecting device could be a T-junction [1], a Y-junction [2], a circulator [3], a manifold [4] or a common resonator [5]. The external junctions utilised in the conventional approach to diplexer design had resulted in more complex and larger size diplexer devices. The complexity of the conventional design is due to the fact that the external junction (or connecting device) needs to be continuously adjusted and optimised in order to achieve a desired result. Also, the large size issue with conventional diplexer is because the connecting device does not contribute to the number of poles contained in the resultant diplexer. To remedy these drawbacks, the work presented in this paper has successfully eliminated the need for an external junction in achieving a microwave diplexer and replacing it with resonators which provide resonant poles in the diplexer response. This paper will show that with a good mastery of the basic principles involved in bandpass filter (BPF) design [6] and dual-band filter (DBF) design [7], engineers can achieve diplexers purely based on existing formulations rather than developing complex optimisation algorithm to achieve the same function. Also, since the resultant diplexer in this paper is formed by coupling a section of the BPF resonators, onto a section of the DBF resonators, a reduced sized diplexer is achieved. This is because the energy distributing resonators (that is, the two DBF resonators), contribute one resonant pole to the diplexer Tx channel and one resonant pole to the diplexer $\mathrm{Rx}$ channel. Therefore, the large size issue with the conventional diplexer design can be avoided, as external junctions (or external/common resonator) are not required.

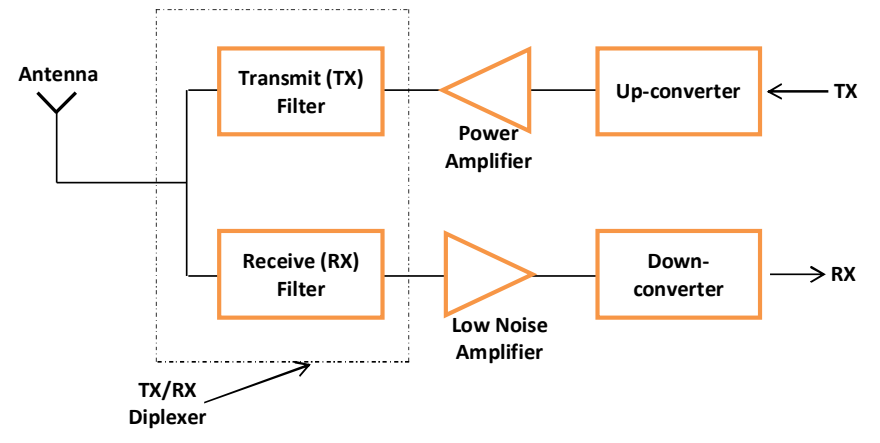

Fig. 1. RF front end of a cellular base station [8]

This new proposed approach to microwave diplexer design involves cascading a section of a DBF, with a section of two BPFs. The dual-band filter resonators present in this proposed diplexer can be viewed as energy distributors which distribute energy towards the Tx and the Rx bands of the diplexer; as they replace the external junctions or the common resonator used in other diplexer designs found in literature. The actual function of the dual-band filter is to establish the two passbands of the diplexer. To verify this design method, a test diplexer is presented with the following specifications: centre frequency, $\mathrm{f}_{0}, 1849 \mathrm{MHz}$; centre frequency of the transmit band, $\mathrm{f}_{0}, \mathrm{TX}, 1800 \mathrm{MHz}$; centre frequency of the receive band, $\mathrm{f}_{\mathrm{O}, \mathrm{RX}}, 1900 \mathrm{MHz}$; fractional bandwidth of the transmit band, $\mathrm{FBW}_{\mathrm{TX}}, 4 \%$; fractional bandwidth of the receive band, $\mathrm{FBW}_{\mathrm{RX}}$, 4\%; passband return loss, RL, $20 \mathrm{~dB}$.

\section{DIPLEXER CIRCUIT MODEL}

To illustrate the novel diplexer design method, a design example with the specification presented in previous section 
will be used to describe the design procedure. The diplexer design is started off by designing two individual 5-pole bandpass filters (BPF1 and BPF2) using the conventional method presented in [6]. BPF1 has a centre frequency corresponding to that of the proposed diplexer transmit band while BPF2 has a centre frequency corresponding to that of the received band. Both channel filters were designed with $20 \mathrm{~dB}$ return loss and $50 \mathrm{Ohms}$ termination. Each has a fractional bandwidth (FBW) of $4 \%$ to match the proposed diplexer transmit and receive bands. The numerical design parameters for both BPF1 and BPF2 are shown in Table1. As explained in [6], L, C and $\mathrm{J}$ are inductance, capacitance and $\mathrm{J}$-inverter values, respectively. $\mathrm{F}$ is the filter centre frequency.

TABLE I. $\quad 5^{\mathrm{TH}}$ ORDER CHEBYSHEV BANSPASS FILTER DESIGN PARAMETERS

\begin{tabular}{|c|c|c|c|c|c|c|}
\hline BPF n & $\begin{array}{c}\text { F n } \\
{[\mathbf{G H z}]}\end{array}$ & $\mathbf{L}[\mathbf{n H}]$ & $\mathbf{C}[\mathbf{p F}]$ & $\mathbf{J n}_{\mathbf{1 1}}$ & $\mathbf{J n}_{\mathbf{1 2}}$ & $\mathbf{J n}_{\mathbf{2 3}}$ \\
\hline 1 & 1.8 & 0.183 & 43.23 & 0.02 & 0.017 & 0.012 \\
\hline 2 & 1.9 & 0.171 & 40.32 & 0.02 & 0.017 & 0.012 \\
\hline
\end{tabular}

A 10-pole dual-band filter (DBF) was also designed using the method presented in [7]. The DBF was designed to operate at the centre frequency of the proposed diplexer, with a combined FBW of $8 \%$ (with equal split of $4 \%$ each, for the upper and the lower passbands). Table 2 shows the numerical design parameters for the DBF.

TABLE II. $10^{\text {TH }}$ ORDER CHEBYSHEV DUAL-BAND BANDPASS FILTER DESIGN PARAMETERS

\begin{tabular}{|c|c|c|c|c|c|c|c|}
\hline $\begin{array}{c}\text { Filte } \\
\mathbf{r}\end{array}$ & $\begin{array}{c}\mathbf{F} \\
{[\mathbf{G H z}]}\end{array}$ & $\mathbf{L}[\mathbf{n H}]$ & $\mathbf{C}[\mathbf{p F}]$ & $\mathbf{J}_{\mathbf{0 1}}$ & $\mathbf{J}_{\mathbf{1 2}}$ & $\mathbf{J}_{\mathbf{2 3}}$ & $\mathbf{J}^{\prime}{ }_{\mathbf{1 1}}$ \\
\hline DBF & 1.85 & 0.354 & 20.90 & 0.02 & 0.017 & 0.012 & 0.013 \\
\hline
\end{tabular}

The diplexer circuit model was established by coupling the first two poles (dualband resonator) of the DBF, onto the last four poles of the transmit channel filter (BPF1) and the last four poles of the receive channel filter (BPF2). Fig. 2 (a), (b), and (c) show the coupling scheme for a conventional diplexer, a common resonator diplexer and the proposed diplexer, respectively. (a)

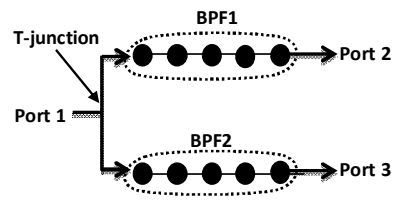

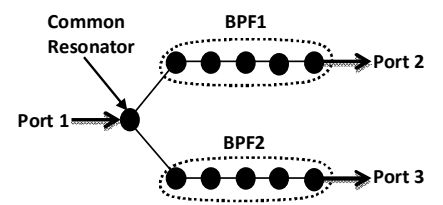

(b)

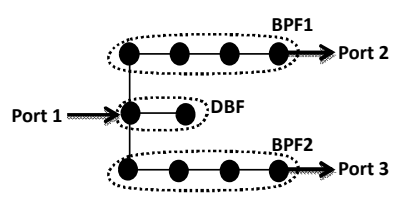

(c)

Fig. 2. 10-pole diplexer structure. (a) Conventional. (b) Common Resonator. (c) Proposed
The proposed diplexer is composed of 10 resonators, making it a $10^{\text {th }}$ order diplexer as shown in Fig. 3. The coupling between resonators in the proposed diplexer circuit model is mainly asymmetrical coupling since the diplexer is made up of three different filters, namely the $\mathrm{DBF}, \mathrm{BPF} 1$ and $\mathrm{BPF} 2$, which all have different centre frequencies as shown in Tables I and II.

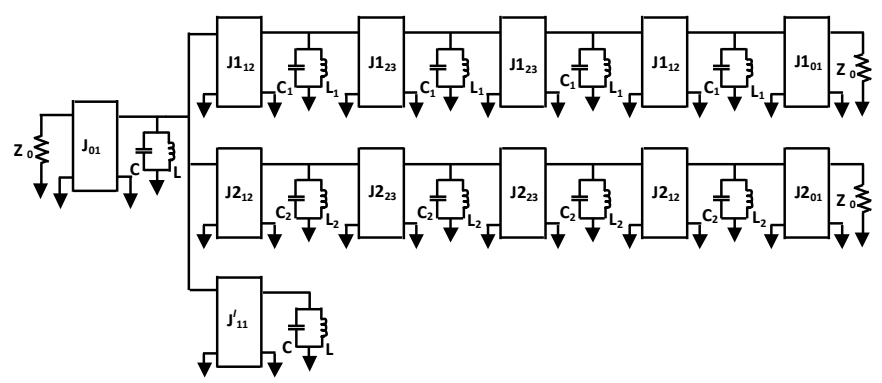

Fig. 3. Diplexer circuit model with identical LC resonators and Jinverters

$$
J^{\prime}{ }_{11}=\sqrt{\frac{C}{L}}\left(\frac{f_{0, R x}^{2}-f_{0, T x}^{2}}{f_{0, R x}^{2}+f_{0, T x}^{2}}\right)
$$

The design parameters for the diplexer are the same as those contained in Tables I and II. J' ${ }_{11}$ is the J-inverter that exists between the two DBF resonators that form the energy distributor for the proposed diplexer. The apostrophe is used here to differential it from the single bandpass filter J-inverter rather than having the mathematically meaning of derivative $\mathrm{J}$ inverter. The numerical value for $\mathrm{J}_{11}{ }_{11}$, as indicated in Table II, is determined using (1) [7], where $f_{0, T X}$ and $f_{o, R X}$ are the centre frequencies for the diplexer transmit and receive bands respectively. The diplexer circuit model was simulated using the Agilent Advanced Design System (ADS) circuit simulator. Before performing the simulation, the couplings between resonators were modelled using the method presented in [9].

The simulation results of the diplexer circuit model are shown in Fig. 4. The results clearly show that the diplexer has a centre frequency of $1.85 \mathrm{GHz}$ as designed. The minimum return loss is approximately $19 \mathrm{~dB}$ across the band. These are in close agreement with the original design specification.

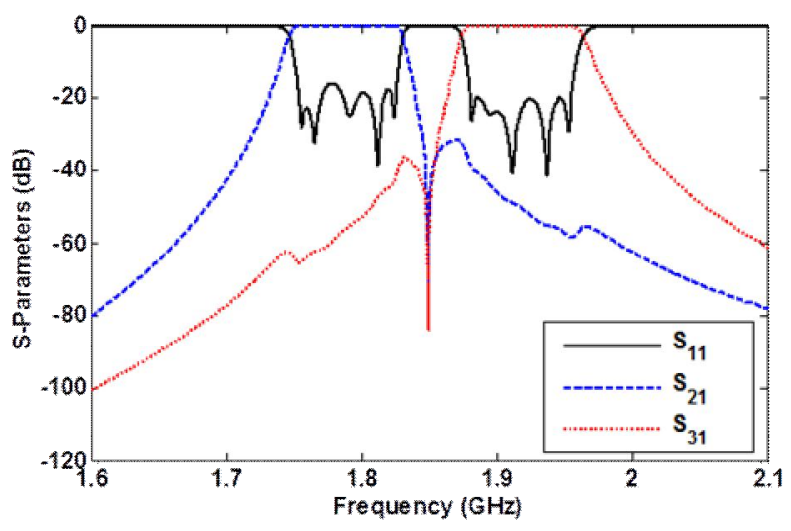

Fig. 4. Simulation responses of the diplexer circuit model 


\section{MICROSTRIP DIPLEXER}

The microstrip square open-loop resonator (SOLR) technique presented in [9] has been utilised in the implementation of the diplexer circuit. The SOLRs utilised in achieving the diplexer presented in this investigation were designed to have the dimensions shown in Fig. 5. The transmit resonator $(\mathrm{Tx})$, the receive resonator $(\mathrm{Rx})$, and the energy distributor resonator (ED), all correspond to the BPF1, the BPF2, and the DBF component filters, respectively. All dimensions were achieved based on the component filters centre frequencies.

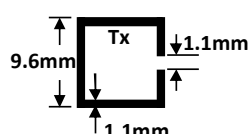

(a)

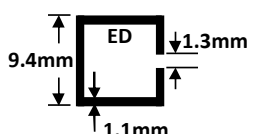

(b)

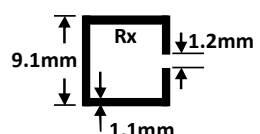

(c)
Fig. 5. Resonator dimensions. (a) Transmit. (b) Energy distributor. (c) Receive.

Using the Agilent ADS Momentum simulator, the coupling coefficients and the external quality factors for the diplexer were determined and presented in Fig. 6 . The coupling between a pair of Tx or Rx resonators (Fig. 6 (a)) where synchronously tuned since they are of equal dimensions. Similarly, the coupling between the ED resonators (Fig. 6 (b)) was also synchronously tuned. On the other hand, the couplings between Tx and ED resonators or Rx and ED resonators (Fig. 6 (c)), were asynchronously tuned because of the variations in the resonator dimensions, i.e. the resonators were resonating at different frequencies. The coupling coefficients of Figs. 6 (a), (b), and (c), were determined from simulating a coupling pair of resonators and using (2) [6], where $f_{1}$ and $f_{2}$ are the eigenmodes from simulating a pair of resonators, $f_{r 1}$ and $f_{r 2}$ are the self-resonant frequencies of resonators 1 and 2, respectively, and $K_{s}$ and $K_{a}$ are for synchronous and asynchronous couplings, respectively.

$$
\begin{aligned}
& K_{s}=\frac{f_{2}^{2}-f_{1}^{2}}{f_{2}^{2}+f_{1}^{2}} \\
& K_{a}=\frac{1}{2}\left(\frac{f_{r 2}}{f_{r 1}}+\frac{f_{r 1}}{f_{r 2}}\right) \sqrt{\left(\frac{f_{2}^{2}-f_{1}^{2}}{f_{2}^{2}+f_{1}^{2}}\right)^{2}-\left(\frac{f_{r 2}^{2}-f_{r 1}^{2}}{f_{r 2}^{2}+f_{r 1}^{2}}\right)^{2}}
\end{aligned}
$$

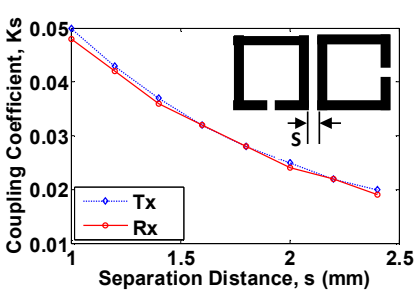

(a)

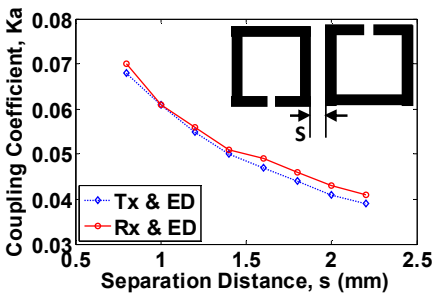

(c)

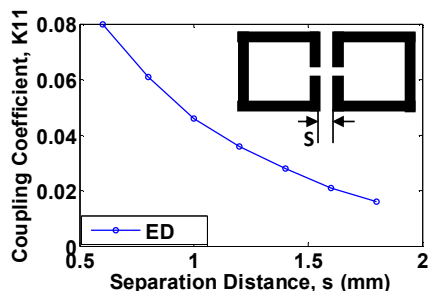

(b)

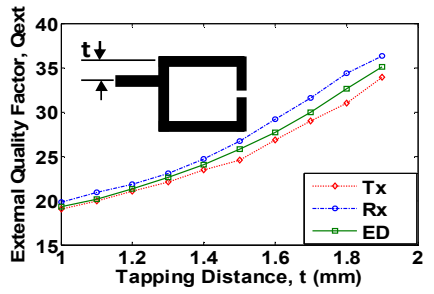

(d)
Fig. 6. Coupling coefficients and external quality factors. (a) $\mathrm{Tx} / \mathrm{Tx}$ and $\mathrm{Rx} / \mathrm{RX}$ couplings. (b) ED/ED coupling. (c) Tx/ED and $\mathrm{Rx} / \mathrm{ED}$ couplings. (d) External quality factor.

Using (2a) and the graph shown in Fig. 6 (a), $\mathrm{S}_{1}, \mathrm{~S}_{2}, \mathrm{~S}_{3}, \mathrm{~S}_{5}$, $\mathrm{S}_{7}, \mathrm{~S}_{8}$ and $\mathrm{S}_{9}$ were achieved. $\mathrm{S}_{4}$ and $\mathrm{S}_{6}$ were also achieved using (2b) and Fig. 6 (c). The diplexer layout, indicating all the desired S-values is shown in Fig. 7.

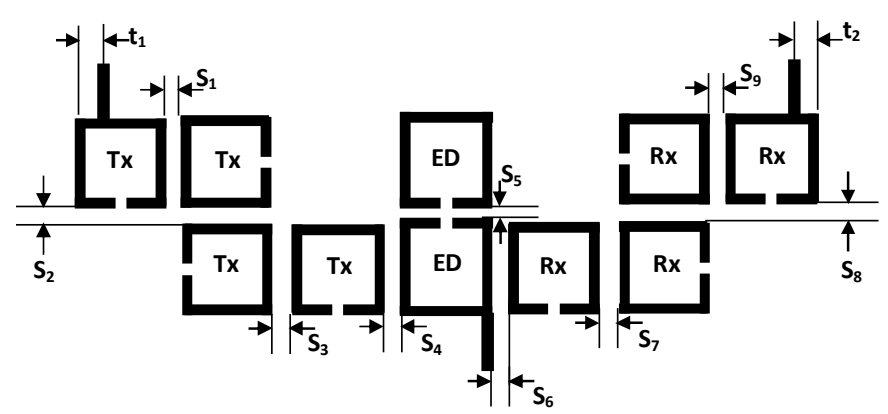

Fig. 7. Diplexer layout $\left(\mathrm{S}_{1}=1.5 \mathrm{~mm}, \mathrm{~S}_{2}=2.0 \mathrm{~mm}, \mathrm{~S}_{3}=2.0 \mathrm{~mm}, \mathrm{~S}_{4}=\right.$ $1.6 \mathrm{~mm}, \mathrm{~S}_{5}=0.9 \mathrm{~mm}, \mathrm{~S}_{6}=1.65 \mathrm{~mm}, \mathrm{~S}_{7}=1.95 \mathrm{~mm}, \mathrm{~S}_{8}=2.0 \mathrm{~mm}, \mathrm{~S}_{9}=$ $1.45 \mathrm{~mm}, \mathrm{t}_{1}=1.45 \mathrm{~mm}, \mathrm{t}_{2}=1.35 \mathrm{~mm}$ ).

The external quality factor, $Q_{\text {ext }}$, was theoretically determined using (3), where $J$ is the J-inverter value between the $50 \mathrm{Ohms}$ feedline and the resonator. Using (3) and applying the specifications for this design, three external quality factor values $24.285,24.285$ and 12.143 for the $\mathrm{Tx}, \mathrm{Rx}$, and $\mathrm{ED}$ resonators of the diplexer were obtained, respectively. These $Q_{\text {ext }}$ values were achieved using the Agilent ADS Momentum simulator by adjusting the tapping distance, $t$, as shown in Fig. 6 (d). The $t$ values that achieved the diplexer specifications are $1.45 \mathrm{~mm}, 1.35 \mathrm{~mm}$, and $0.0 \mathrm{~mm}$ for $\mathrm{Tx}, \mathrm{Rx}$, and $\mathrm{ED}$ resonators, respectively.

$$
Q_{\text {ext }}=\frac{\omega_{0} C}{J}
$$




\section{SimUlATION AND MEASUREMENT}

Electromagnetic (EM) simulation was carried out using the Agilent ADS Momentum simulator. Conductor and dielectric loss parameters were included in the simulation. The RT/Duroid 6010LM substrate with a dielectric constant of 10.8 , a loss tangent of 0.0023 , and a substrate thickness of 1.27 $\mathrm{mm}$ was used for the simulation. A copper conductor with thickness of 16 micron $(\mu \mathrm{m})$ and conductivity, $\sigma=5.8 \times 10^{7}$ $\mathrm{S} / \mathrm{m}$ was assumed for both the top and bottom metals of the microstrip. However, surface roughness and thickness variation of the substrate material were not considered.

The microstrip diplexer was fabricated using the same material employed in the EM simulation. The fabrication was based on printed circuit board (PCB) milling process. The photograph of the microstrip diplexer is shown in Fig. 8. In order to facilitate measurement of the diplexer, three SMA (Sub-Miniature version A) connectors were fitted onto the three input/output ports as shown. The testing and measurement was carried out using the Agilent Vector Network Analyzer. Fig. 9 shows the measured results indicating that an isolation $\left(\mathrm{S}_{32}\right)$ of $50 \mathrm{~dB}$ was achieved between the transmit $\left(\mathrm{S}_{21}\right)$ and the receive $\left(\mathrm{S}_{31}\right)$ bands. The measured minimum insertion loss of the transmit band is 2.88 $\mathrm{dB}$, while that of the receive band is $2.95 \mathrm{~dB}$.

The measured results and the EM loss simulation results are both presented in Fig. 10 for ease of comparison. The graphs clearly show a good agreement between the simulation and measurement. The additional transmission zeros shown in Fig. 10 is the result of unwanted cross-couplings between non adjacent resonators which is an artefact.

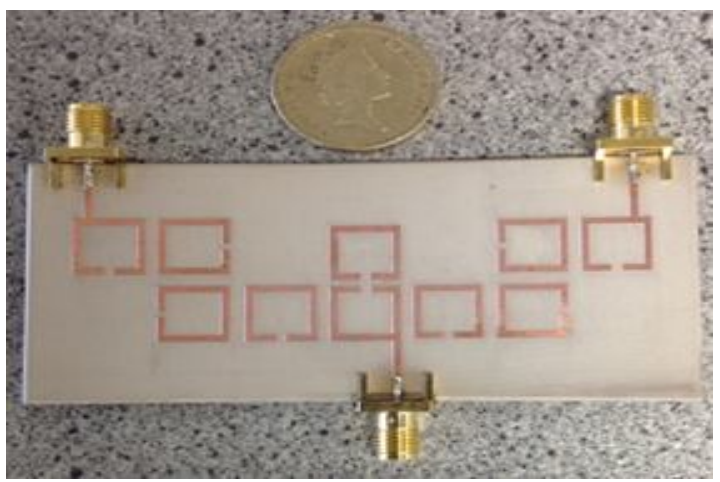

Fig. 8. Photograph of the fabricated microwave diplexer

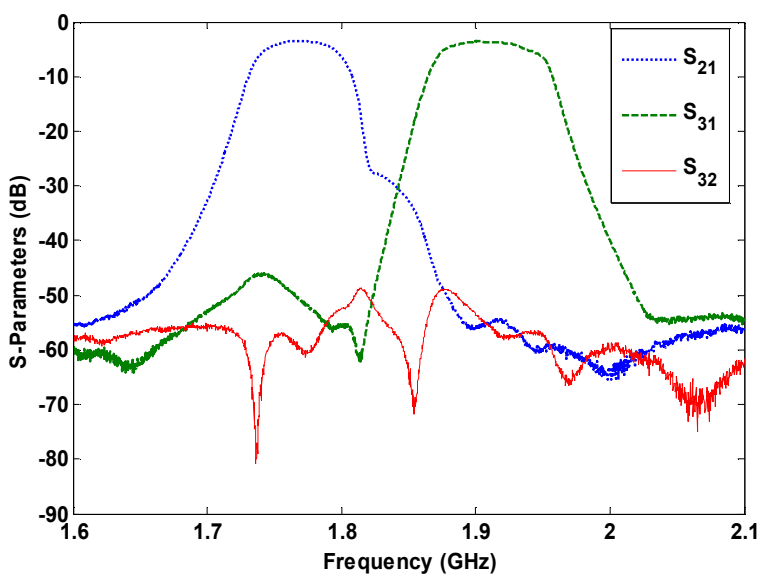

Fig. 9. Measured result of the microwave diplexer

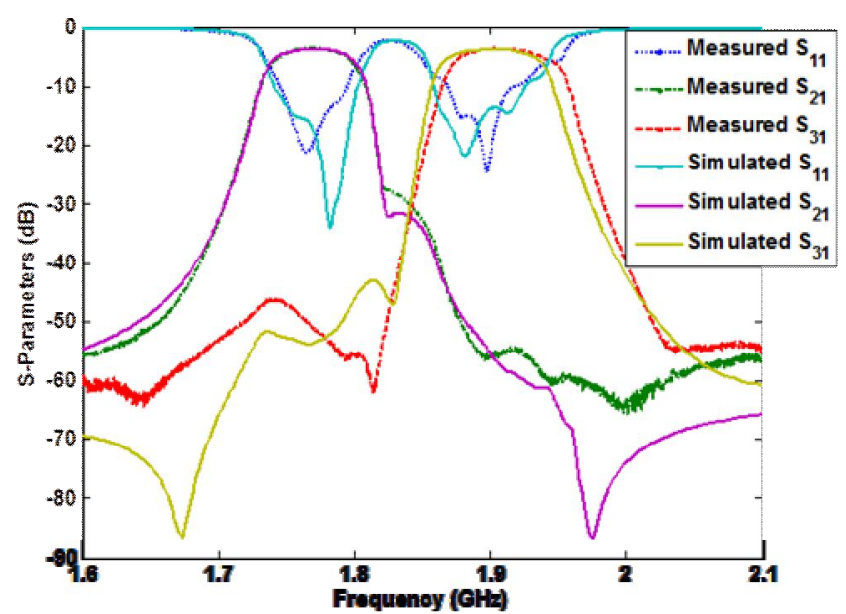

Fig. 10. Comparison of the simulated and measured diplexer results

\section{CONCLUSION}

A microwave diplexer achieved by coupling a dual-band bandpass filter with two single-bands bandpass filters has been presented. The design has been experimentally validated and the performance of the diplexer presented. Measured results indicated that the isolation between the Tx and $\mathrm{Rx}$ bands is about $50 \mathrm{~dB}$, which is very encouraging. The measured minimum insertion loss of the Tx and the $\mathrm{Rx}$ bands are $2.88 \mathrm{~dB}$ and $2.95 \mathrm{~dB}$, respectively. As a result of the very good bands isolation of the diplexer, only a very small amount of signal is expected to deflect into the wrong direction. The simulated and measured results show good agreement.

\section{REFERENCES}

[1] S. H. Yun, M. S. Uhm, and I. B. Yom, "Design of multiplication free high power Ka-band diplexer with an E-plane T-junction," IEEE AsiaPacific Conference on Communications, Perth, 3-5 October, 2005, pp. 582-585. doi:10.1109/APCC.2005.1554128

[2] S. Bastioli, L. Marcaccioli, and R. Sorrentino, "An original resonant Yjunction for compact waveguide diplexers," IEEE MTT-S Int. Microwavs Symp. Dig., pp. 1233-1236, June 2009.

[3] T. Kodera, and C. Caloz, "Integrated leaky-wave antennaduplexer/diplexer using CRLH uniform ferrite-loaded open waveguide," IEEE Trans. Microwave Antennas Propag., vol. 58, no. 8, pp. 25082514, August 2010.

[4] M. Guglielmi, "Optimum CAD procedure for manifold diplexers," IEEE MTT-S Int. Microwave Symp. Dig., pp. 1081-1084, June 1993.

[5] R. Wang, and J. Xu, "Synthesis and design of microwave diplexers with a common resonator junction," IEEE Int. Conf. on Microwave and Millimeter Wave Tech. (ICMMT), vol. 2, pp. 1-4, 2012.

[6] J.-S. Hong, Microstrip Filters for RF/Microwave Applications, New York: Wiley, 2011.

[7] K. S. K. Yeo and A. O. Nwajana, "A novel microstrip dual-band bandpass filter using dual-mode square patch resonators," Progress in Electromagnetic Research C, vol. 36, pp. 233-247, 2013.

[8] I. C. Hunter, L. Billonet, B. Jarry and P. Guillon, "Microwave filtersapplications and technology," IEEE Trans. Microw. Theory Techn., vol. 50, no. 3, pp. 794-805, Mar. 2002.

[9] J.-S. Hong and M J. Lancaster, "Coupling of microstrip square openloop resonators for cross-coupled planar microwave filters," IEEE Trans. Microwave Theory Techn., vol. 44, no. 12, pp. 2099-2109, December 1996. 